\title{
ONE SIDED INVERTIBILITY AND LOCALISATION II
}

\author{
by C. R. HAJARNAVIS
}

(Received 9 March, 1993)

1. Introduction. The aim of this paper is to generalise the results of [7] from the prime to the semiprime case. It was shown, for instance, that if $M$ is the annihilator of a simple right module $S$ of projective dimension 1 over a Noetherian prime polynomial identity (PI) ring $R$ then $M$ is either an invertible ideal or an idempotent ideal [7, Proposition 4.2]. One of the main applications of this result was that a prime Noetherian affine PI ring of global dimension less than or equal to 2 is a finite module over its centre. It turns out that this theorem is valid more generally when the ring is semiprime [1, Theorem A]. Clearly this requires [7, Proposition 4.2] also to be strengthened to the semiprime case. We do this by showing that a right invertible maximal ideal in a semiprime Noetherian PI ring is also left invertible (Theorem 3.5).

We also show that a right invertible prime ideal in a semiprime Noetherian PI ring is localisable (on both sides), (Theorem 4.3). In order to do this we need to prove the theorem first for maximal ideals using the Artin-Rees property and then appeal to a result of Stafford on the localisability of cliques in polynomial extensions. This route seems surprisingly intricate but perhaps some difficulty is to be anticipated given that it is usually not straightforward to change sides.

2. Preliminaries and notation. We refer the reader to [7, Section 2] for the notation which we shall continue to follow. However we need to make some changes to allow for zero divisors and torsion.

Let $R$ be a ring with a quotient ring $Q$ and let $I$ be an ideal of $R$. The sets $I^{*}=\{q \in Q \mid q I \subseteq R\}$ and $I^{\#}=\{q \in Q \mid I q \subseteq R\}$ will only be defined when $I$ contains a regular element of $R$. In particular a right (left) invertible ideal will by definition be assumed to contain a regular element.

Let $\mathscr{S}$ be a multiplicatively closed subset of $R$. The set $\mathscr{S}$ is called a right Ore set if given $a \in R$ and $c \in \mathscr{S}$ there exist $a_{1} \in R$ and $c_{1} \in \mathscr{S}$ such that $a c_{1}=c a_{1}$.

Let $\mathscr{S}$ be a right Ore set. Then $T=\{x \in R \mid x c=0$ for some $c \in \mathscr{P}\}$ is an ideal of $R$. When $R$ is right Noetherian, the images in $R / T$ of the elements of $\mathscr{S}$ are regular elements of $R / T$ [5, Proposition 9.9]. So in this case we can form the right localisation $R_{\mathscr{S}}$ which in reality is an overring of $R / T$.

A left Ore set is defined analogously. When $R$ is a Noetherian ring and $\mathscr{S}$ is an Ore set on both sides, by [5, Proposition 9.9] the ideal $T$ defined above coincides with $T^{\prime}=\{x \in R \mid c x=0$ for some $c \in \mathscr{S}\}$. Thus $R_{\mathcal{S}^{p}}$ can be considered a two sided localisation in this case. In particular if $P$ is a prime ideal of $R$ and $S=\mathscr{C}(P)$ is an Ore set then $P$ is said to be localisable. In this case $R_{\mathscr{S}}$ is a local ring and is denoted by $R_{P}$.

More generally, the term localisable is also applicable to a clique of linked prime ideals $\left\{P_{\alpha}\right\}$ where the Ore set considered is $\cap \mathscr{C}\left(P_{\alpha}\right)$. For the definitions and further detail in this area we refer the reader to [5].

An ideal $I$ is said to have the right Artin-Rees property (AR property) if given a right ideal $E$ there exists an integer $n \geq 1$ such that $E \cap I^{n} \subseteq E I$.

Glasgow Math. J. 37 (1995) 15-19. 
$Q(A)$ will denote the quotient ring of a ring $A$ when it exists. Let $R$ be a semiprime Noetherian ring and $P_{1}, \ldots, P_{n}$ the minimal primes in $R$. Since the $P_{i}$ are annihilators we have $\mathscr{C}(0) \subseteq \mathscr{C}\left(P_{1} \cap \ldots \cap P_{s}\right)$ for each $s$. It is easily seen then that there is a natural homomorphism from $Q(R)$ into the ring $Q\left(R /\left(P_{1} \cap \ldots \cap P_{s}\right)\right)$. It follows from this that if $I$ is a right invertible ideal of $R$ then the image of $I$ is a right invertible ideal of the ring $R /\left(P_{1} \cap \ldots \cap P_{s}\right)$.

$A(R)$ will denote the Artinian radical of a ring $R$.

$R$ is called a pri ( $p l i)$ ring if every right (left) ideal of $R$ is principal.

As before, unless otherwise stated, conditions will be assumed to hold on both right and left.

For any unexplained terminology we refer the reader to [3] or [5].

\section{Right invertible maximal ideals.}

Lemma 3.1. Let $M$ be a right invertible maximal ideal of $R$ where $R$ is a semiprime Noetherian PI ring. Then $M$ has the AR property (on both sides) in $R$.

Proof. Let $P_{1}, \ldots, P_{n}$ be the minimal primes in $R$. The proof is by induction on $n$. For $n=1$ this is the prime case. [7, Proposition 4.1(i)] shows that $M$ is invertible. The AR property follows by [3, Lemma 3.3].

Assume now that the result holds in the ring $R /\left(P_{1} \cap \ldots \cap P_{n-1}\right)$. As stated in Section 2 the images of $M$ are right invertible ideals in the rings $R /\left(P_{1} \cap \ldots \cap P_{n-1}\right)$ and $R / P_{n}$ respectively. By the induction hypothesis (or trivially), $\left[M+\left(P_{1} \cap \ldots \cap P_{n-1}\right)\right]$ / $\left(P_{1} \cap \ldots \cap P_{n-1}\right)$ has the AR property on both sides. Again by the prime case the same also applies to the ideal $\left[M+P_{n}\right] / P_{n}$. We shall now show that this gives the AR property for $M$ in $R$.

Let $E$ be a right ideal of $R$. Then there exists an integer $k \geq 1$ such that

$$
\left(E \cap P_{n}\right) \cap M^{k} \subseteq\left(E \cap P_{n}\right) M+\left(P_{1} \cap \ldots \cap P_{n-1}\right) .
$$

Therefore

(i) $\left(E \cap P_{n}\right) \cap M^{k} \subseteq\left(E \cap P_{n}\right) M$ since $P_{1} \cap \ldots \cap P_{n-1} \cap P_{n}=0$.

(ii) Also there exists an integer $s \geq 1$ such that $E \cap M^{s} \subseteq E M^{k}+P_{n}$.

Clearly we may take $s \geq k$. Then (ii) gives

(iii) $E \cap M^{s} \subseteq E M^{k}+\left(E \cap M^{k}\right) \cap P_{n}$.

(i) and (iii) now yield $E \cap M^{s} \subseteq E M^{k}+\left(E \cap P_{n}\right) M \subseteq E M$.

Thus $M$ has the right AR property. The left AR property follows analogously.

COROLlary 3.2. With $R$ and $M$ as above, $\mathscr{C}(M)$ is an Ore set.

Proof. This now follows from Lemma 3.1 and a result of P. Smith [3, Lemma 11.9] noting that the factor rings $R / M^{n}$ are Artinian.

Lemma 3.3. Let $R$ be a semiprime Noetherian local PI ring. Suppose that $J$ the Jacobson radical of $R$ is right invertible. Then $R$ is a prime pri and pli ring. In particular, $J$ is an invertible ideal.

Proof. By Jategaonkar's theorem [3, Theorem 7.11] we have $\bigcap_{n=1}^{\infty} J^{n}=0$. By Formanek's theorem every non-zero ideal of $R$ contains a non-zero central element. Let $a$ 
be a non-zero central element of $R$. Then there exists an integer $k \geq 1$ such that $a \in J^{k}$ but $a \notin J^{k+1}$. Therefore $a\left(J^{\#}\right)^{k} \subseteq R$ and $a\left(J^{\#}\right)^{k}$ is an ideal of $R$. If $a\left(J^{\#}\right)^{k} \subseteq R$ then $a\left(J^{\#}\right)^{k} \subseteq J$. Since $a$ is central, this implies that $a \in J^{k+1}$ which is a contradiction. Hence $a\left(J^{*}\right)^{k}=R$ and so $a R=J^{k}$. Since $J$ is right invertible, $\ell(J)=0$. So $a$ is regular and $a R$ is an invertible ideal. Hence $J$ is invertible. We have also shown that every non-zero ideal of $R$ contains a regular element. So $R$ must be a prime ring. By [6, Proposition 1.3] it follows that $R$ is a pri and pli ring.

We note that if $P$ is a prime ideal of a Noetherian ring and $\mathscr{f}$ is an Ore set then either $\mathscr{S} \subseteq \mathscr{C}(P)$ or $\mathscr{S} \cap P \neq \varnothing$.

Proposition 3.4. Let $P$ be a right invertible and localisable prime ideal of a semiprime Noetherian PI ring $R$. Let $P_{1}, \ldots, P_{n}$ be the minimal prime ideals of $R$ numbered so that precisely $P_{1}, \ldots, P_{k}$ lie inside $P, 1 \leq k \leq n$. Denote $\mathscr{I}=\mathscr{C}(P) \cap \mathscr{C}\left(P_{k+1}\right) \cap \ldots \cap \mathscr{C}\left(P_{n}\right)$. Then $\mathscr{S} \subseteq \mathscr{C}(0), \mathscr{S}$ is an Ore set and $P R_{\mathscr{S}}$ is principal as a right as well as a left ideal of $R$.

Proof. Since $\mathscr{C}(P)$ and each $\mathscr{C}\left(P_{i}\right)$ are Ore sets, we obtain by [3, Lemma 13.4] that $\mathscr{S}$ is an Ore set. As above $\mathscr{C}(P) \subseteq \mathscr{C}\left(P_{i}\right)$ for $1 \leq i \leq k$. So $\mathscr{S} \subseteq \mathscr{C}(0)$. Thus the localisation $R_{\mathscr{S}}$ exists and is a semiprime partial quotient ring of $R$. By [3, Theorem 1.23] $R_{\mathscr{S}}$ splits as a direct sum of rings $R_{y}=B \oplus C$ where $A(B)=0$ and $C$ is Artinian. So by [3, Lemma 4.10] no minimal prime of $B$ is maximal. Therefore $B \cap P_{i} R_{\mathscr{S}}=B$ for $k+1 \leq i \leq n$. Also since $P$ properly contains a minimal prime ideal, we have $C \cap P R_{\mathscr{S}}=C$. Thus $B \cap P R_{\mathscr{S}}$ is the unique maximal ideal of $B$ and $B$ is a semiprime Noetherian local PI ring. So by Lemma 3.3, $B \cap P R_{\mathscr{S}}$ is a principal right as well as left ideal of $B$. Hence the same is true of $P R_{\mathscr{S}}$ as an ideal of $R_{y}$.

REMARK. Theorem 4.3 will show that the assumption concerning the localisability of $P$ in the above is in fact redundant.

THEOREM 3.5. Let $M$ be a right invertible maximal ideal of a semiprime Noetherian PI ring. Then $M$ is left invertible.

Proof. We follow the notation of Proposition 3.4 with $M=P$. By Corollary 3.2, $M$ is localisable. So we have $M R_{\mathscr{f}}=m R_{\mathscr{S}}$ for some $m \in M$. Since $M$ is right invertible, so is $M R_{\mathscr{S}}=R_{\mathscr{S}} M$ (noting that $\mathscr{S} \subseteq \mathscr{C}(0)$ and so $R_{\mathscr{y}}$ is an overring of $R$ ). Hence $\ell\left(M R_{\mathscr{S}}\right)=0$ and $m$ is a regular element. The rest of the proof can now proceed as in [4, Lemma 4.1].

We can now generalise [7, Proposition 4.1] to the semiprime case.

THEOREM 3.6. Let $S$ be a simple right module of projective dimension 1 over a semiprime Noetherian PI ring $R$. Let $M$ be the annihilator of $S$ in $R$. Then $M$ is either an invertible or an idempotent ideal.

Proof. Since $R$ is a PI ring $M$ is a maximal ideal and $R / M$ is isomorphic to a finite number of copies of $S$. Thus $M$ is projective as a right ideal. We have $M \subseteq M^{*} M \subseteq R$ and so $M=M^{*} M$ or $M^{*} M=R$. The former possibility implies that $M$ is idempotent and the latter that $M$ is invertible by Theorem 3.5 . 
As a consequence of Theorem 3.5 we also have the following result.

COROLLARY 3.7. Let $R$ be a semiprime Noetherian PI ring. Let $M$ be a maximal ideal of $R$ which is not minimal. Then $M$ is a principal right ideal $\Leftrightarrow M$ is a principal left ideal.

Proof. Let $M=R a$ with $a \in M$. By assumption $a$ is a regular element and so $M$ is right invertible. The result now easily follows.

4. Right invertible prime ideals. We can now extend Corollary 3.2 from the maximal to the prime case.

Lemma 4.1. Let $\mathscr{S}$ be a right Ore set in a semiprime left Noetherian ring $R$. Then the right localisation $R_{\mathscr{S}}$ is a semiprime ring.

Proof. The set $T=\{x \in R \mid x c=0$ for some $c \in \mathscr{P}\}$ is an ideal and we must show that it is semiprime. Let $A$ be an ideal of $R$ such that $A^{2} \subseteq T$. Since $R$ is left Noetherian ${ }_{R} T$ is finitely generated and there exists $c \in \mathscr{S}$ such that $T c=0$. Hence $A^{2} c=0$ and so $A(A c)=0$. Since $R$ is semiprime, we have $A c A=0$. Thus $(A c)^{2}=0$ and $A c=0$ again since $R$ is semiprime. Therefore $A \subseteq T$ and $T$ is a semiprime ideal as required.

COROLlary 4.2. Let $R, \mathscr{S}$ be as above and let I be a right invertible ideal of $R$. Then $I R_{\mathscr{S}}$ is a right invertible ideal of $R_{\mathscr{S}}$.

Proof. It is easily seen that $T$ is an annihilator. So $\mathscr{C}(0) \subseteq \mathscr{C}(T)$. Thus there is a natural homomorphism from $Q(R)$ into $Q\left(R_{\mathscr{S}}\right)$. It follows that $I R_{\mathscr{S}}$ is a right invertible ideal of $R_{\mathscr{y}}$.

Since it is not known if cliques in a Noetherian PI ring are localisable, we need to shift to the polynomal ring $R[x]$ in the next theorem.

THEOREM 4.3. Let $P$ be a right invertible prime ideal in a semiprime Noetherian PI ring. Then $P$ is localisable.

Proof. The polynomial ring $R[x]$ is a semiprime Noetherian PI ring and $P[x]$ is a prime ideal of $R[x]$. So by [9] the clique of $P[x]$ is localisable in $R[x]$. Clearly $P[x]$ is a right invertible prime ideal of $R[x]$. Let $S=\bigcap \mathscr{C}\left(P_{i}\right)$ where $P_{i}$ ranges over the clique of $P[x]$. Let $T=\{r \in R[x] \mid r c=0$ for some $c \in \mathscr{S}\}$. Thus $T$ is an ideal of $R[x]$ and as explained in Section 2, $T$ coincides with its left hand analogue. By Lemma 4.1 $T$ is a semiprime ideal and by Corollary $4.2 P[x] / T$ is a right invertible prime ideal of the semiprime Noetherian PI ring $R[x] / T$. Now $P[x] / T$ extends to a right invertible maximal ideal in the localisation of $R[x] / T$ at the Ore set $(\mathscr{Y}+T) / T$. So by Corollary 3.2 , this extension of $P[x] / T$ is a localisable maximal ideal. It follows that $P[x] / T$ is localisable in $R[x] / T$. Hence $P[x]$ is localisable in $R[x]$. Thus the clique of $P[x]$ is a singleton. Therefore the clique of $P$ in $R$ is a singleton and so by [5, Theorem 12.21] $P$ is localisable in $R$.

Remark. An elementary proof along the lines of [7, Theorem 3.5] can be given to show directly that $P$ is right localisable. Then one approach to deduce left localisation is to generalise [2, Theorem A] to the semiprime case. This can be done by using the methods of this paper to obtain a version of the Artin-Rees property on the left. 
An immediate application of the above is the following theorem on unique factorisation rings. Recall that $R$ is a right unique factorisation ring (right UFR) if every height 1 prime ideal of $R$ is principal as a right ideal.

THEOREM 4.4. Let $R$ be a semiprime Noetherian PI ring which is a left UFR. Then $R$ is a maximal order and hence a direct sum of prime rings.

Proof. We shall merely sketch the modifications required to the proof of $[\mathbf{7}$, Lemma 3.3]. Without loss of generality, we may assume that $R$ is an indecomposable ring. By [3, Theorem 1.23] we may also assume that $A(R)=0$. Then no minimal prime of $R$ is maximal. Let $P$ be a height 1 prime. Then $P$ is generated by a regular element. Let $P_{1}, \ldots, P_{n}$ be the minimal primes of $R$ numbered so that exactly $P_{1}, \ldots, P_{k}$ lie in $P, k \leq n$. Let $\mathscr{S}=\mathscr{C}(P) \cap \mathscr{C}\left(P_{k+1}\right) \cap \ldots \cap \mathscr{C}\left(P_{n}\right)$. By Theorem 4.3, $\mathscr{C}(P)$ is an Ore set and hence by Proposition 3.4 so is $\mathscr{Y}$. As in [7, Lemma 3.3] we can show that $R=\cap R_{\mathscr{S}}$ where $\mathscr{S}$ is constructed as above for each height 1 prime ideal of $R$. Lemma 3.3 and the proof of Proposition 3.4 now show that $R_{\mathscr{y}}$ is a direct sum of a semisimple Artinian ring and a pri and pli ring. In particular $R_{S}$ is a maximal order. It follows that $R$ is a maximal order.

The last part of the theorem follows from [8, Lemma 5.2].

Corollary 4.5. Let $R$ be a semiprime Noetherian PI ring. Then $R$ is right $U F R \Leftrightarrow R$ is left UFR.

Proof. This now follows from the above and [7, Theorem 4.4].

Finally the ring of $2 \times 2$ upper triangular matrices over the integers shows that the results of this paper such as Theorem 3.5 do not generalise to the non-semiprime case even when the ring has an Artinian quotient ring.

\section{REFERENCES}

1. A. Braun and C. R. Hajarnavis, Finitely generated PI rings of global dimension 2, to appear in J. Algebra.

2. A. Braun and R. B. Warfield Jr., Symmetry and localisation in Noetherian prime PI rings, $J$. Algebra 118 (1988), 322-334.

3. A. W. Chatters and C. R. Hajarnavis, Rings with chain conditions, Research Notes in Mathematics 44 (Pitman, 1980).

4. A. W. Chatters and C. R. Hajarnavis, Ideal arithmetic in Noetherian PI rings, J. Algebra 122 (1989), 475-480.

5. K. R. Goodearl and R. B. Warfield Jr., An introduction to noncommutative Noetherian rings, London Mathematical Society Student Texts 16 (Cambridge University Press, 1989).

6. C. R. Hajarnavis and T. H. Lenagan, Localisation in Asano orders, J. Algebra 21 (1972), $441-449$.

7. C. R. Hajaranavis, One sided invertibility and localisation, Glasgow Math. J. 34 (1992), 333-339.

8. J. C. Robson, Non-commutative Dedekind rings, J. Algebra 9 (1968), 249-265.

9. J. T. Stafford, unpublished.

Mathematics Institute

UNIVERSTTY OF WARWICK

COVENTRY CV4 7AL 